\title{
Local field enhancement of nano-structured metallic target irradiated by polarized laser beam
}

\author{
M. Nikbakht \\ mnik@znu.ac.ir
}

\section{H. Mahdieh}

\author{
Department of Physics, Iran University of Science and Technology, Narmak, Tehran, Iran \\ present address: Department of Physics, University of Zanjan, P.O.Box 45196-313, Zanjan, Iran \\ Department of Physics, Iran University of Science and Technology, Narmak, Tehran, Iran
}

The local field enhancement is studied numerically in samples of metallic nanoparticles (NPS) randomly distributed over a metallic substrate. The sample was assumed to be irradiated by polarized laser beam. Based on dipole-dipole approximation (DDA), the electric field was calculated Two-dimensionally at the irradiated region. The results show that the optimized field enhancement is strongly depends on NPs characteristics, beam polarization and incident angle.

[DOI: http://dx.doi.org/10.2971/jeos.2012.12025]

Keywords: field enhancement, polarization, surface plasmons

\section{INTRODUCTION}

The study of strong enhancements of electromagnetic fields has been of interest for its wide range of applications in nonlinear optics, photochemistry and light-matter interactions [1]. These fluctuations have been investigated extensively in complex targets like metal fractals and metal-dielectric composites; see for example [2] and references there in. It has been found that the areas of large field fluctuations are localized in different small parts of a target that change with the wavelength. Some of these targets contain metallic NPs that are characterized by their size, shape and dielectric constant $[3,4]$. These NPs can cause a local change in the refractive index of an interface between two different materials, leading to an effective change in reflection [5, 6]. Recently, several studies have been devoted in this aspect to improve the light coupling into solar cells [7]-[9], controlling the in-plane substrate resonance feature by the substrate material $[10,11]$, or to enhance the transparency in antireflective structures by using random distribution of nanoholes [12]. Nanostructured surfaces are also interested as highly absorbing targets for highpeak-power subpicosecond laser-matter interaction [13, 14]. The strong fluctuations of electric field across such targets results in corresponding enhancement of nonlinear effects. Efficient production of hot electrons with moderate laser intensity has already attracted studies in utilization of porous targets to get an increase in hard and soft X-ray yields $[15,16]$.

In laser interacting complex targets, the maximum field enhancement can be achieved by optimization of both the macroscopic parameters of electromagnetic wave and the nanostructure characteristics. Many of these characteristics have been studied since now [17]-[20]. Among them, optimizing illumination conditions can be considered as an important issue affecting the performance of the experimental setup. The illumination conditions include the state of polarization of the incident beam, the angle of incidence, and parameters of the laser beam. While the role of the incident beam polarization is more or less clear, less attention was paid to the effect of the incident angle on the field enhancement.

In spite of big efforts in field enhancement in semicontinious metal film, less attention has paid on NPs density. In reference [21] we studied the optical properties of fractal aggregates of metal NPs (Immobilized to an Au substrate). The average density of particles in fractals is much smaller than in bulk materials and tends to zero with increasing the fractal size. The main result was that the aggregation of initially isolated particles into fractals results in enhancements of the responses and existence of an optimum value for cluster size which maximizes the averaged local field enhancement (for p-polarized incident beam). To see the contribution of NPs density to the enhancement factors of SERS, the calculation performed for random distribution of metal NPs.

The objective of the present work is to study the local field enhancement of randomly distributed NPs over a gold substrate. The effect of oblique polarized incident beam as well as the NPs filling factor on the averaged local field enhancement are reported. It is shown that the field enhancement and the optimum angle of incidence are correlated to the NPs filling factor.

\section{THEORY AND MODEL}

The DDA method is an extensively used model for the numerical calculations of magnitude and spatial distribution of the local electric field of metallic NPs with sizes smaller than $100 \mathrm{~nm}$. We assumed that metallic NPs are placed randomly over a flat metallic substrate with filling factor $\mathrm{f}$. Such metal 
(a)

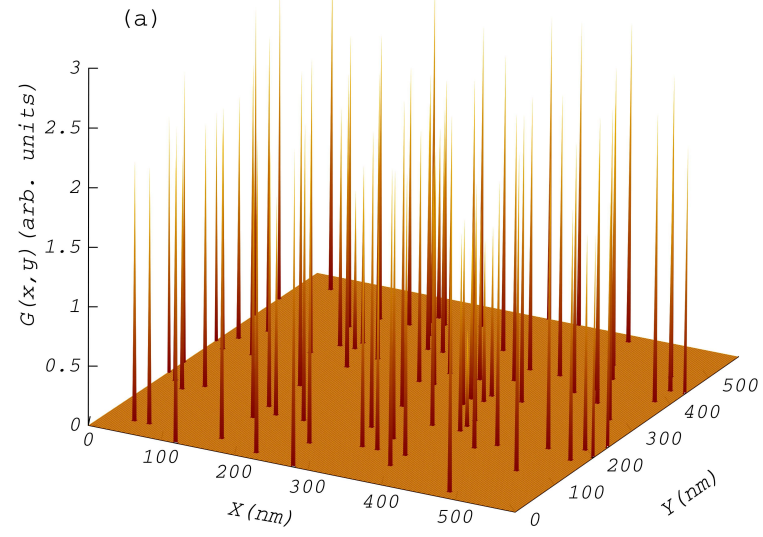

(c)

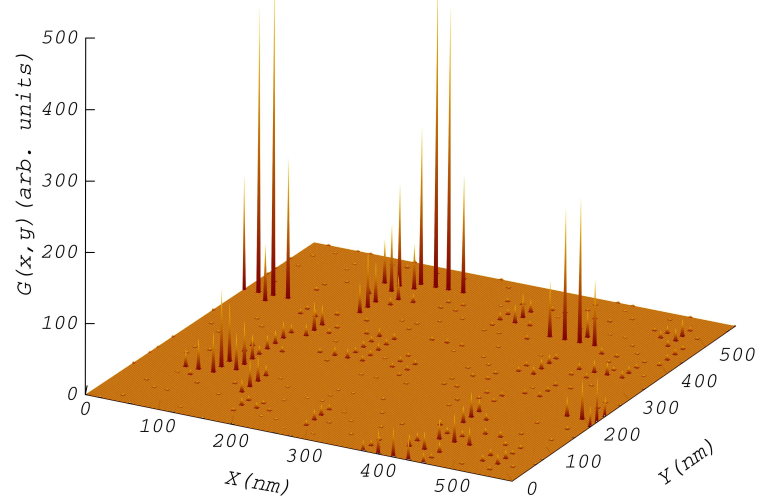

(b)

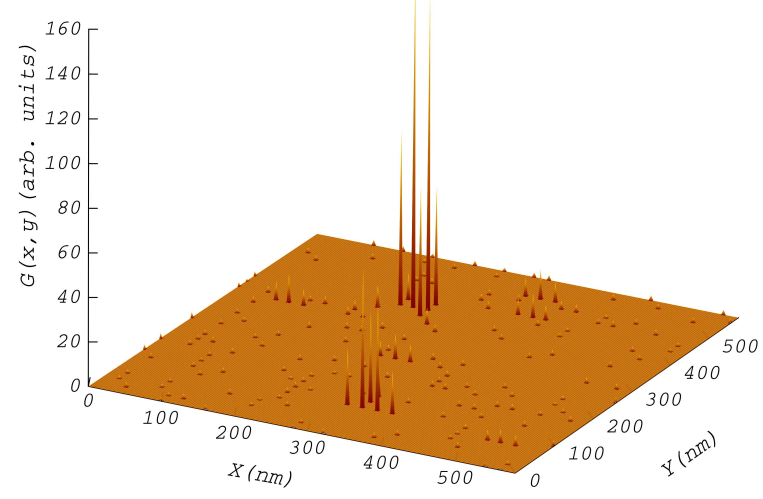

(d)

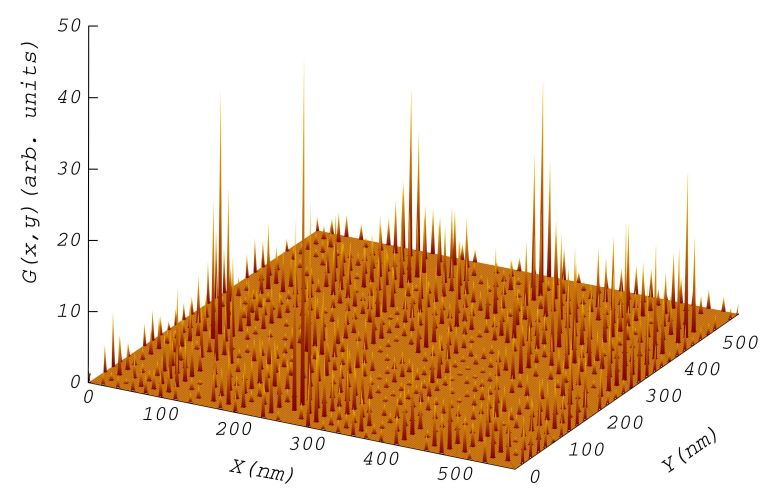

FIG. 1 Distribution of the normalized local field intensities $G(x, y)=|E(x, y)|^{2} /\left|E_{\text {inc }}\right|^{2}$ of Au nano-particles located on Au substrate for incident P polarized beam $(\theta=63$ degree) at different filling factors $f$. (a) $f=0.1$, (b) $f=0.2$, (c) $f=0.35$, and (d) $f=0.8$.

nanostructured target was assumed to be exposed obliquely to a laser beam with specific polarization. The filling factor is related to the NPs concentration, which reflects the ratio of filled sites by NPs with respect to the total available sites on the substrate. The enhancement of electric field is due to electromagnetic interactions between NPs (assuming as radiating dipoles) and the substrate. In the Rayleigh limit and for a spherical particle, it can be assumed that only the dipole surface-plasmon resonance contributes to the enhancement process [22]. With this assumption, it is possible to consider each NP as an elementary dipole and introduce corresponding interaction operators. In the presence of an applied electromagnetic, dipole-dipole interaction occurs. According to the macroscopic Maxwell equations and surface boundary conditions, another type of electromagnetic effect is the change in incoming and outgoing fields at the surface of the substrate which is the exerted field on NPs.

The dipole moment $\mathbf{d}$ of NPs in the proximity of a surface can be expressed as:

$$
\mathbf{d}=\alpha \mathbf{E}_{0}+\alpha \hat{\mathbf{W}} \mathbf{d}
$$

Where $\alpha$ is NPs polarizability, $\mathbf{E}_{0}$ is the exerted field at the position of NP and $\hat{\mathbf{W}}$ is dipole-dipole interaction tensor. In the situation under study, the field acting on the dipoles (NPs position) consists of two contributions. The direct incident field coming from the external plane wave $\left(\mathbf{E}_{i n c}\right)$ plus the reflected field from the substrate surface $\left(\mathbf{E}_{r}\right)$. Considering the electric field intensity and the phase of the incident beam to be known, the amplitude and phase shift of the reflected field are calculated using the well-known Fresnel formula and GoosHanchen shift $[23,24]$. By performing a series of careful calculations and using DDA method to calculate the dipole moments [21], the local fields on NPs can be obtained from:

$$
\mathbf{E}_{\text {local }}=\alpha^{-1} \mathbf{d}
$$

In this work, The NPs were assumed to be distributed randomly on a square lattice with lattice constant $a=20 \mathrm{~nm}$. Spatial distributions of dimensionless enhanced local field $G(x, y)=E^{2}{ }_{\text {local }} / E^{2}{ }_{\text {inc }}$, and the spatially averaged enhanced local field (over the illuminated area) of the NPs i.e $\langle G\rangle$, were calculated for inclined incident beam with two polarizations $\mathrm{S}$ and $\mathrm{P}$. In these calculations, targets with different filling factors were considered. The calculations were also conducted for the incident beam with different incident angles $\theta$. The dispersive dielectric response of gold was calculated as described in Ref. [25].

\section{RESULTS AND DISCUSIONS}

Figure 1 shows the local field enhancement at the irradiated target surface for $\mathrm{P}$ polarization. The figure shows the calculation results for different filling factors $\mathrm{f}=0.1,0.2,0.35$, and 0.8 which are presented in (a), (b), (c), and (d), respectively. In this figure, the incident beam wavelength is assumed to be $\lambda=800 \mathrm{~nm}$ and the incident angle typically fixed at 63 degree. As it can be seen, the field is enhanced more effectively with increasing the filling factor, but further increase in $\mathrm{f}$ results in 

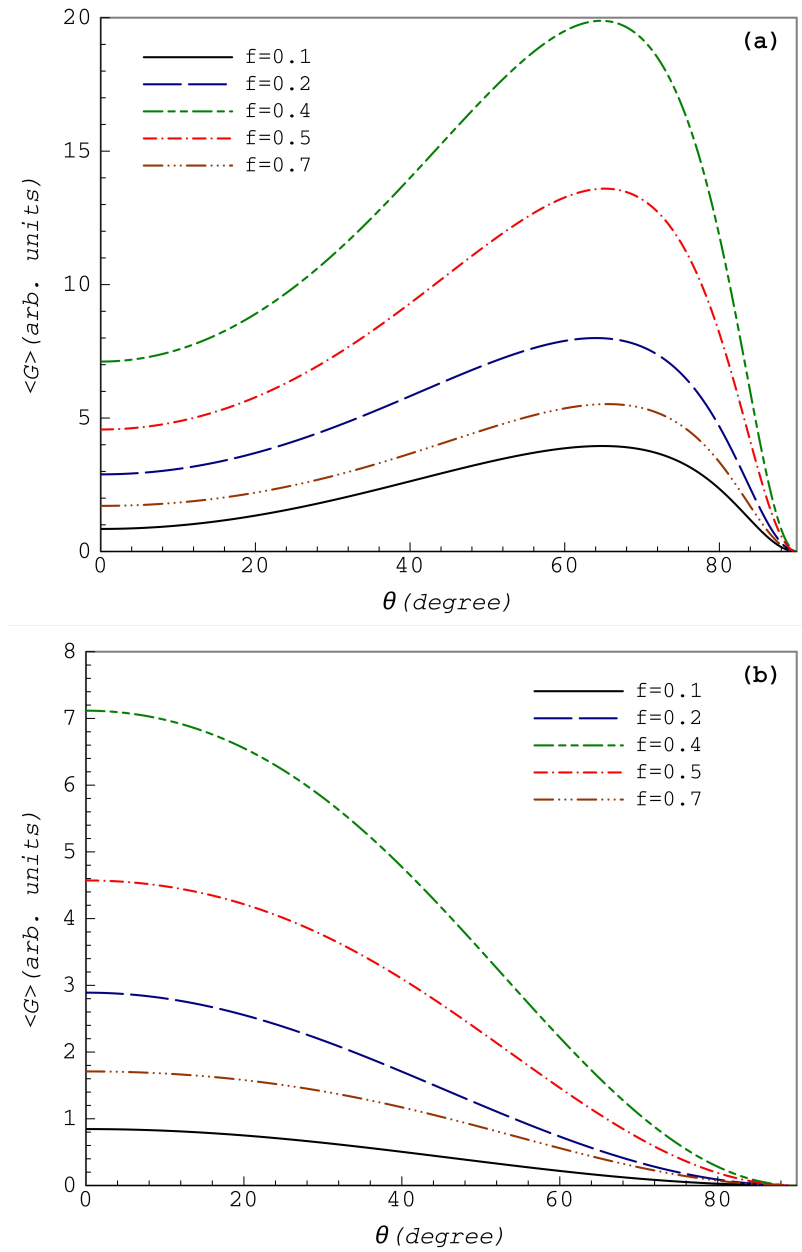

FIC. 2 Averaged local field enhancement as a function of incident beam's angle for different filling factors of nano-particles $(f=0.1 ; f=0.2 ; f=0.4 ; f=0.5$; and $f=0.7$ ); (a) For $P$ polarized incident beam, (b) For $S$ polarized incident beam.

decreasing the enhancement effects. Increasing the field intensities as well as peak spatial distribution by $\mathrm{f}$, leads to an increase in the averaged enhancement of the field intensity.

Figures 2(a) and 2(b) show the averaged local field enhancement at the irradiated target surface for $\mathrm{P}$ and $\mathrm{S}$ polarizations, respectively. The results are presented as a function of incident beam angle for different NPs filling factor. As Figure 2(a) shows, for $\mathrm{P}$ polarization, the averaged local field enhancement increases with incident angle and reaches to a maximum at $\theta \approx 63$ degree and then decreases for higher angles. However, Figure 2(b) shows somehow different behavior for $\mathrm{S}$ polarization. In this case, the averaged local field enhancement decreases with increasing the incident angle. This is obvious since both the incident and the reflected (S polarized) beams do not meet the phase-matching at the substrate surface. From Figure 1, it is clear that, the values for field enhancements are gigantically large. However, since the large spikes in these figures spread in small area (in comparison with the illuminated area), averaging the field over the space results in small values for $\langle G\rangle$ in Figure 2(a).

It is also clear that for a given value of NPs filling factor, only the $P$ polarized beam has an optimum angle which maximizes the average field enhancement. In limiting values, ( $f \Rightarrow 0$ and $\mathrm{f} \Rightarrow 1$ ), the sample behaves similar to a bulk metallic surface

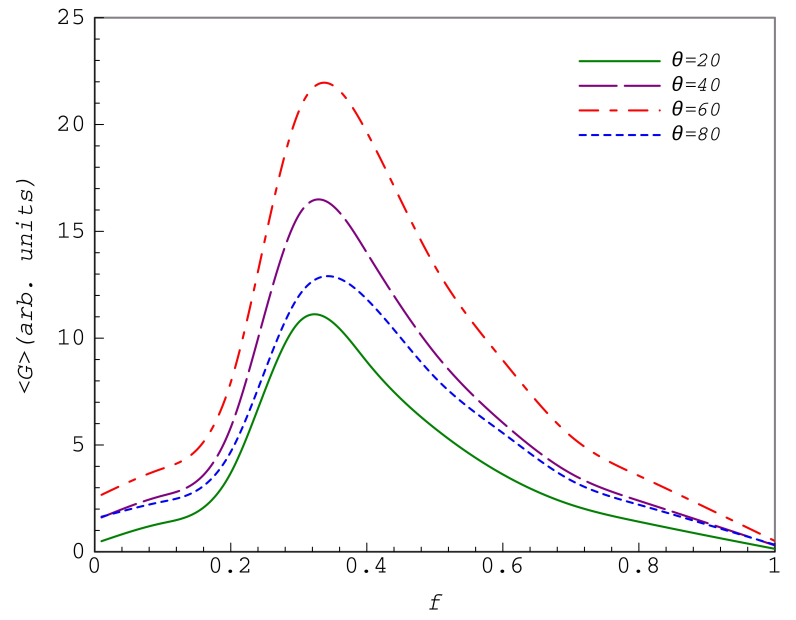

FIG. 3 Averaged local field enhancement plotted as a function of filling factor for $P$ polarized beam with different incident angles.

and also averaged local field (and optimum value of incident angle for P polarized beam) become the same as those that can be obtained from Fresnel relations. However, for intermediate values of NPs concentration, increasing filling factor from $\mathrm{f} \sim 0$ to some critical value $\left(\mathrm{f}_{c} \sim 0.35\right)$, results in enhancing average local field for both polarization at all incident angles. This is obvious since the near field coupling increases due to the decrease in spacing between NPs. Such enhancement depends strongly on the incident beam angle.

For more details, the averaged local field enhancement is presented in Figure 3 as a function of NPs filling factor for P polarized wave. The figure shows the results for different incident angles. It can be seen that the optimum value of filling factor that maximizes $\langle G\rangle$ occurs at $\mathrm{f} \sim 0.35$ for almost all incident angles. The results also show that the maximum value of $\langle G\rangle$ increases with increasing the incident angle, and reaches to a peak value and then decreases for higher incident angles. Therefore, there would be an optimum value for both of incident angle and the filling factor in which the value of $\langle G\rangle$ is maximized. The calculations show that $\langle G\rangle$ is maximized at incident angle of $\theta \sim 63$ degree for almost all filling factor values.

Figure 4 shows an optimum angle (which maximizes $\langle G\rangle$ for $P$ polarized beam) as a function of NPs filling factors. The maximum value of $\langle G\rangle$ varies with changing $f$ and incident angle. As the diagram shows, the local field can effectively be maximized for an incident angle of $\theta \sim 63$ degree with a small deviation ( $\Delta \theta \sim 3$ degree).

\section{CONCLUSION}

In conclusion, the local field enhancement of nanostructured target that was assumed to be exposed to an oblique polarized laser beam investigated numerically. The target was assumed to consist metallic NPs distributed randomly over a metallic substrate. We perform a systematic study for different value of filling fractions covering the whole range, from the extremedilute to the closed-packed limits. From the results it was concluded that the field enhancement strongly depends on the 


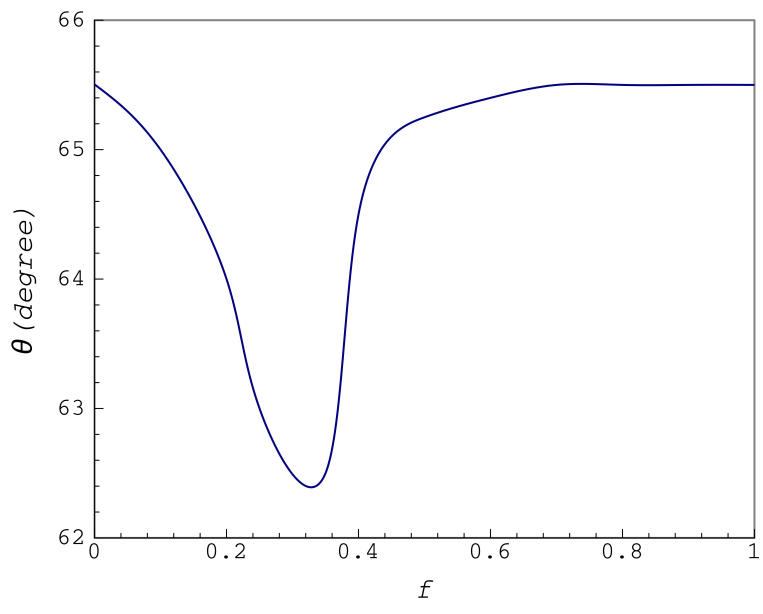

FIG. 4 Optimum angle as a function of filling factors for $\mathrm{P}$ polarized incident beam which maximizes the averaged local field.

incident beam angle, polarization, and also NPs filling factor. According to the results, for the laser beam with a specific polarization and particular angle of incidence, there exists a critical value for NPs filling factor $\left(\mathrm{f}_{\mathcal{C}} \sim 0.35\right)$ which maximizes the average field enhancement. It was also shown that, as long as NPs filling factor has an important effect in field enhancement, both incident beam angle and polarization play more important role in oblique illumination. Due to continuity of field at the substrate surface (boundary conditions), average local field enhancement has a peak in a particular incident angle for $\mathrm{P}$ polarized beam while it is a decreasing function of angle for $S$ polarized beam. According to the results, maximum field enhancement can be achieved by $P$ polarized beam with incident angle around critical angle $\theta \sim 63$ degree which can be tuned slightly by NPs filling factor.

\section{References}

[1] S. Eustis, and M. A. El-Sayed, "Why gold nanoparticles are more precious than pretty gold: Noble metal surface plasmon resonance and its enhancement of the radiative and nonradiative properties of nanocrystals of different shapes," Chem. Soc. Rev. 35, 209-217 (2006).

[2] A. K. Sarychev, and V. M. Shalaev, "Electromagnetic field fluctuations and optical nonlinearities in metal-dielectric composits," Phys. Rep. 335, 275-371 (2000).

[3] G. K. Stamplecoskie, J. C. Scaiano, V. S. Tiwari, and H. Anis, “Optimal Size of Silver Nanoparticles for Surface-Enhanced Raman Spectroscopy," J. Phys. Chem. C 115, 1403-1409 (2011).

[4] K. L. Kelly, E. Coronado, L. L. Zhao, and G. C. Schatz, "The Optical Properties of Metal Nanoparticles: The Influence of Size, Shape, and Dielectric Environment," J. Phys. Chem. B 107, 668-677 (2003).

[5] L. A. Lyon, M. D. Musick, P. C. Smith, B. D. Reiss, D. J. Pena, and M. J. Natan, "Surface plasmon resonance of colloidal Au-modified gold films," Sensor. Actuat. B 54, 118-124 (1999).

[6] S. L. Diedenhofen, G. Vecchi, R. E. Algra, A. Hartsuiker, 0. L. Muskens, G. Immink, E. P. A. M. Bakkers, et.al, “Broad-band and omnidirectional antireflection coatings based on semiconductor nanorods," Adv. Mater. 21, 973-978 (2009).

[7] S. D. Standridge, G. C. Schatz, and J. T. Hupp, "Distance Dependence of Plasmon-Enhanced Photocurrent in Dye-Sensitized Solar Cells,"
J. Am. Chem. Soc. 131, 8407-8409 (2009).

[8] S. Tsai, M. Ballarotto, D. B. Romero, W. N. Herman, H. Kan, and R. J. Phaneuf, "Effect of gold nanopillar arrays on the absorption spectrum of a bulk heterojunction organic solar cell," Opt. Express 18, A528-A535 (2010).

[9] I. Diukman, L. Tzabari, N. Berkovitch, N. Tessler, and M. Orenstein, "Controlling absorption enhancement in organic photovoltaic cells by patterning $\mathrm{Au}$ nano disks within the active layer," Opt. Express 19, A64-A71 (2011).

[10] C. Chen, J. Wang, F. Tsai, Y. Lu, Y. Kiang, and C. C. Yang, "Fabrication of sphere-like Au nanoparticles on substrate with laser irradiation and their polarized localized surface plasmon behaviors," Opt. Express 17, 14186-14198 (2009).

[11] Y. Tanaka, G. Obara, A. Zenidaka, N. N. Nedyalkov, M. Terakawa, and M. Obara, "Near-field interaction of two-dimensional highpermittivity spherical particle arrays on substrate in the Mie resonance scattering domain," Opt. Express 18, 27226-27237 (2010).

[12] J. Son, L. K. Verma, A. J. Danner, C. S. Bhatia, and H. Yang, "Enhancement of optical transmission with random nanohole structures," Opt. Express 19, A35-A40 (2010).

[13] C. Reich, P. Gibbon, I. Uschmann, and E. Forster, "Yield Optimization and Time Structure of Femtosecond Laser Plasma K $\alpha$ Sources," Phys. Rev. Lett. 84, 4846-4849 (2000).

[14] P. Gibbon and 0. N. Rosmej, "Stability of nanostructure targets irradiated by high intensity laser pulses," Plasma. Phys. Contr. F. 49, 1873-1883 (2007).

[15] M. H. Mahdieh, R. Fazeli, and G. J. Tallents, "Soft x-ray enhancement from a porous nano-layer on metal targets irradiated by long laser pulses," J. Phys. B-At. Mol. Opt. 42, 125602 (2009).

[16] P. Gibbon, M. Masek, U. Teubner, W. Lu, M. Nicoul, U. Shymanovich, A. Tarasevitch, P. Zhou, K. Sokolowski-Tinten, and D. V. der Linde, "Modelling and optimisation of fs laser-produced K $\alpha$ sources," Appl. Phys. A-Mater. 96, 23-31 (2009).

[17] W. A. Tisdale, K. J. Williams, B. A. Timp, D. J. Norris, E. S. Aydil, and X. Zhu, "Hot-Electron Transfer from Semiconductor Nanocrystals," Science 328, 1543-1547 (2010).

[18] J. Li, S. Chen, P. Yu, H. Cheng, W. Zhou, and J. Tian, "Large enhancement and uniform distribution of optical near field through combining periodic bowtie nanoantenna with rectangular nanoaperture array," Opt. Lett. 36, 4014-4016 (2011).

[19] J. Laverdant, S. Buil, J. P. Hermier, and X. Quelin, "Near-field intensity correlations on nanoscaled random silver-dielectric films," J. Nanophotonics 4, 049505 (2010).

[20] J. Sancho-Parramon, "Near-field coupling of metal nanoparticles under tightly focused illumination," Opt. Lett. 363527 (2011).

[21] M. Nikbakht and M. H. Mahdieh, "Optical responses of gold nanoparticles undergoing a change to cluster aggregates and laser beam characteristics effect," J. Phys. Chem. C 115, 1561-1568 (2011).

[22] V. A. Markel, V. M. Shalaev, E. B. Stechel, W. Kim, and R. L. Armstrong, "Small-particle composites. I. Linear optical properties," Phys. Rev. B 53, 2425-2436 (1996).

[23] M. Born, and E. Wolf, Principles of Optics (Pergamon Press, Oxford, 1987).

[24] W. J. Wild, and C. L. Giles, "Goos-Hanchen shift from absorbing media," Phys. Rev. A 25, 2099-2101 (1982).

[25] A. D. Rakic, A. B. Djurisic, J. M. Elazar, and M. L. Majewski, “Optical properties of metallic films for vertical-cavity optoelectronic devices," Appl. Optics 37, 5271-5283 (1998). 